\title{
Conceptual Adequacy in Legal Translation
}

\author{
Nare Chobanyan \\ Yerevan State University
}

\begin{abstract}
The aim of the present article is to provide an overview of the main difficulties encountered by legal translators, and work out some practical solutions so that the translator could provide an adequate translation in compliance with the norms of the target legal system. Legal translations raise very complex theoretical and practical problems and, therefore, an interdisciplinary comparative approach to the two legal systems and languages should be manifested by specialized translators. This study demonstrates that despite the common assumption that legal translations are literal, they may be translated differently depending on the context and aim of its translation. When translating a legal document, one is thus faced with the challenge of providing a translation that makes a legal as well as linguistic sense. Consequently, a translator can provide an accurate translation only if he/she has an understanding of the SL and the TL legal systems.
\end{abstract}

Key words: comparative linguistics, comparative law, SL and TL legal systems, culture-specific elements, paradigms of translation, court system, legal institutions, conceptual adequacy.

\section{Introduction}

The legal systems of different countries are based on different legal traditions and cultural principles. This leads to legal terminology varying from country to country and remaining specific to each society. The very specific jargons of the legal texts are as complicated as mathematic equations. There is no room for literal translation, and the task of the translator is to fully understand the legal systems and language-specific details the two countries possess. 
In fact, an overabundance of resources found on the Internet today may lead to confusion and make inexperienced translators focus on tools rather than content. Therefore, translators should be equipped with reliable resources and be taught how to grasp the meaning in context of legal terms, as well as how to find appropriate equivalents in the target language.

In the modern globalized world, translation lacks depth in theorization. Paradigm shifts in translation theory occur when the changes in the social, economic, cultural and religious environments lead to significant changes in the practice of translation and that these changes then can no longer be adequately theorized by the old paradigms of translation. In order to provide an adequate interpretation, description, explanation and prediction of the impact of globalization on the theory and practice of translation, it is worth emphasizing that globalization has changed the mainstream of translation practice from canonical translation to professional (i.e. non-canonical) translation. Translation theories based on comparative literary study or on linguistics fail to provide an objective and comprehensive theoretical framework for the mainstream practice of translation. Influenced by theories of the knowledge-driven economy, translation should now serve the demand of the global economy and the global community.

The process of translation is a process of decision-making. It is a set of procedures and strategies for making judgments when selecting the optimal choice from a range of potential equivalents. The theory of translation should attempt to understand how that decision-making is accomplished. It should also explicate how the professional translator moves from the concrete source text to producing the most appropriate target text.

\section{Paradigms of Translation}

According to Anthony Pym, Plurality of Paradigms tends to have many valuable ways of approaching translation. The true stuff of translation theory is the body of practice, what translators actually do and how translation users react to what they do. Rather, we would recognize that there is a translation reality which is extremely diverse and which calls for different translation responses. 
As Pym notes, for a translator who intends to work in the field of law, the problem of correctly identifying the English equivalent of the Armenian legal term and vice versa is often a difficult task. A good translation is the one which has the same impact on the target language audience as the original text has on the source language audience. Therefore, Pym states two related questions the translator always has to bear in mind: Who is the intended audience? What is the purpose of the text? The practice of translating seems to follow the modern translation theory that advocates the priority of the purpose as a decisive factor. If the translation is required only for an informal purpose (e.g. the business partner mainly needs to be informed on certain legal matters), then the translator may choose a simplified version, and sometimes it is the target reader that asks for it in order to fully understand the message. On the other hand, the translation can be required to be submitted as evidence in a court of law, or may represent a document that is to comply with legal requirements - for instance, a contract or a power of attorney. In this case the translation becomes even more difficult and the translator has to connect the source and target languages so as to meet the requirements of a fully functional translation (Pym 2012:21-46).

Dating back to the wars between Egypt and Mesopotamia, the two dominant rivals of the early Eurasian world, the oldest known recorded evidence of legal translation is the Egyptian-Hittite Peace Treaty of 1271 B.C. For over 2000 years, general translation studies were dominated by the debate whether a translation should be literal or free. Since both legal and religious texts are normative and cultural-bound, it is not surprising, that the early history of legal translation is most closely related to that of Bible translation, which needs "strict literal" translation. In the seventeenth century the French churchman and scholar Pierre Daniel Huet raised his voice and rejected strict literal translation as "primitive", insisting that interlinear translation requires no intellect on the part of the translator. In his opinion, the translator must respect the basic rules of grammar and syntax in the target language, yet not 'adulterate' the source text by producing a free translation. Thus, Huet advocated a "refined" form of literal translation in which the words are translated in context, not in isolation (Šarčević 2000:293). In the field of law, it was the 
practitioners who finally raised the question whether legal translations must follow the letter of the source text, as was traditionally believed, or whether they can be written in the spirit of the target language.

After the so-called "cultural turn" in the 1980s, when factors other than purely linguistic ones began to be taken into account, translation strategies shifted according to the purpose of the translation (Vermeer 1996:41-42). Other scholars would rather describe this approach as taking the particular communicative situation into account. As Šarčević rightly argues, this approach cannot be applied to legal translations without restriction, because these texts are subject to special rules that govern their use in the mechanism of the law. When translating legislative texts, the translator will usually adhere closely to the wording of the source text. For example, when it comes to certificates and diplomas, with the culture-specific elements widely used, the aim of which is the delivery of personal information, its recognition and application, the strategy is generally to retain original forms without cultural adaptation. This normally means using the simplest translation equivalents as suggested by dictionaries, glossaries, encyclopedias or other credible sources. Moreover, some elements must also be left untouched (e.g. addresses, names of people and places). Borrowings, calques, naturalizations or descriptive translations are commonly used, because the first and most critical rule in the translation of such texts (apart from being communicative) is the retention of the source cultural elements. This is done with official names of countries, institutions, job titles, and the forenames of the holders of the documents. We also retain the sense (or names) of administrative units, the names of normative acts governing the formats of the documents if they are mentioned, and the references to all forms of proof of authenticity stamps, watermarks etc. (Šarčević 2000:277-293).

According to Correia, when it comes to the names of institutions, it is sometimes difficult to find a foreign language equivalent in accessible sources like dictionaries, glossaries, or corpora. If the institution itself does not suggest the officially established and acceptable version of its name, the only solution is to borrow the original name and add the translator's version of descriptive translation, or sometimes to calque (the only excuse for using the name of an 
equivalent institution in the target culture is the situation in which the functions of both institutions are similar and there is no better solution). However, we must remember that the translation is introduced after the first appearance of the original term for informative purposes, as the most appropriate strategy is still to use the original versions of such names (Correia 2003:38).

\section{The Principle of Fidelity in Legal Translation}

After all, the principle of fidelity in legal translations represents only one of the challenges for the translator. Guralnik (1979), in Webster's English Dictionary, writes that faithfulness/fidelity means "the quality of being accurate, reliable, and exact." Fidelity in translation is passing of the message from one language into another by producing the same effect in the other language, (in sense and in form), in a way that the reader of the translation would react exactly as the reader of the original text. The relationship of fidelity between the original and its translation has always preoccupied translators, but the problem is, as far as translation is concerned, one should decide to whom, to what the supposed fidelity pertains.

The language system itself with its syntactic and semantic implications places certain demands on the translator and even creates limits for the translation. The following are some of the areas which can give rise to errors:

\section{Polysemantic and homonymic words}

Many words have a number of meanings, either related to each other in the event of polysemy, or looking alike simply by coincidence (homonyms). A translator may fail to notice that the word which seems familiar has a meaning different from the ones which it has in other typical contexts, or simply be unable to choose the best meaning from those listed in a dictionary.

For instance, the word title (Yn\nuL) has a number of meanings in English, but is hardly ever used in the legal sphere - for that purpose the word degree is used (polysemantic word). 


\section{Synonyms and collocations}

Synonymous expressions cannot always replace each other in any context, but tend to be distinguished by different lexes or form different collocations with other words. A subversive activity may be illegal, but not illegitimate or lawless, a child may be legitimate or illegitimate, but not illegal, illicit, or unlawful. A husband will not refer to his wife as legitimate, or legal, but as lawful.

Apart from the linguistic issues, one of the central challenges, which the translators of legal texts face, is the ability to fully understand the requirements of various legal systems worldwide. In this respect, comparative law plays an important role in legal translation, as it allows for the identification of similarities and differences among legal systems.

While the practice of legal translation requires an excellent knowledge of comparative law for the linguistic transfer to be successful, educational institutions do not usually train their students in how to make the most of comparative law in the translation of legal texts or how to rationally solve the problems arising from the differences that inevitably exist between legal systems.

Adhering to the pragmatic-functionalist approach by Weston, the primary function of legislative texts is regulatory (prescriptive) and as such these texts differ from other LSP texts. When it comes to understanding a foreign legal system, this implies that the translator should at least have some basic knowledge of the other legal system and legal culture. Legal system and legal culture are inherently interwoven, the difference being that the study of legal culture takes as an axiom the fact that two societies can have similar legal systems in some formal sense and yet different systems in terms of living law or actual practice. From this it follows that the translator must be aware of the most obvious similarities and differences between the systems and cultures being analyzed (Weston 1991:23).

Of vital importance to translation is ensuring that the legal effects are the same in both the source language and culture and the target language and culture. To do this, the translator partially applies the method of legal 
comparison, e.g. when comparing the court system or other legal institutions, such as marriage, in two different legal systems (e.g. Armenia's and Canada's legal systems). The Armenian court system differs considerably from the Canadian system due to the fact that Armenia is a unitary state (a state governed as a single power in which the central government is ultimately supreme) while Canada is a federal state (a political entity characterized by a union of partially self-governing states or regions under a central (federal) government). In the institution of marriage, for instance, the same-sex marriages are not recognized in the Armenian legal system in contrast to the Canadian one. In this respect, the translation of some culture-bound legal concepts referring to the institution of marriage may become a challenge for the translator. In the source text (in the Canadian legal system) we find, for instance, the pivotal concepts of co-mother and co-motherhood. These terms were recently introduced to cover the concepts arising from the new form of parenthood in a same-sex marriage of two women: co-mother (the legal parent of her female partner's child) and co-motherhood (being the legal parent of her female partner's child). To find out whether there is comparability in the target legal culture, the Armenian and Canadian legal cultures must be compared with respect to the regulation of the same-sex relationships. The Armenian legal system does not allow same-sex marriages, whereas the Canadian legal system does. Consequently, there are no legal concepts similar to the English comother and co-motherhood, and the translator has to apply an appropriate strategy to cover the linguistic and legal gap. There are several legal terms in English referring to the above-discussed legal concept - "joint mother", "comother", "joint status as mother", "joint maternity", "co-motherhood" and "comaternity". Here the prefix $c o$ - and the adjective joint convey the idea of sharing, and the translator may choose to use their Armenian equivalents (huulu-, huuuunti) to convey those terms into Armenian if there are no

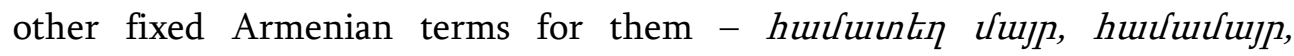

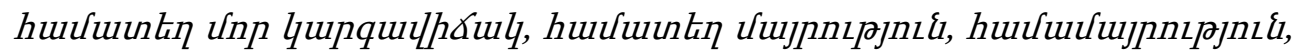
etc. Here the translator works out an adequate translation for the given terms applying the transformational approach either at the morphological (in case of 
the prefix $c o^{-}$) or lexical level (in case of the word joint).

Thus, for an equivalent legal translation, a number of strategies should be applied in the form of shifts and transformations to ensure appropriateness, that is, the potential reader should get the same information not necessarily rendered by the same kind of linguistic means. For example, when translating verdicts the English present tense in The Court rules as follows... is adequately rendered into Armenian by means of the past tense Ruunupuin y lankg... In other contexts, however, a similar change in tense might be inappropriate.

\section{Conceptual Adequacy in Legal Translation}

Legal translation leaves little margin to subjectivity. It rather depends on legal conditions, comparative law and legal interpretation rules to achieve accuracy and conceptual adequacy. In a major proportion of legal translation, this is a matter of legal consistency, and hence of conformity to legal sources and discourses, rather than a matter of individual preferences. Conceptual adequacy implies that the terminology used should cover the same semantic areas - this is sometimes difficult because the systems of law are not mirror images of each other. In one country, for example, the terms infant, toddler, baby, child, teenager, underage, minor, juvenile, adolescent, etc., refer to age groups which do not necessarily overlap with the particular age groups in another language. Though most countries set the age of maturity at 18 , there are different ages as well, such as age of sexual consent, marriageable age, school leaving age, drinking age, driving age, voting age, smoking age, etc. Although a person may attain the age of maturity in a particular jurisdiction, they may still be subject to age-based restrictions regarding matters, such as the right to vote or stand for elective office, act as a judge, and many others. There are even countries, where age of adulthood refers only for male or female only, such as in Iran, the adulthood age is 15 for males and in Pakistan 16 for females only. For example, the following article from the RA Civil Code (www.arlis.am) may cause some misunderstanding if translated into English without specifying the age of adulthood mentioned in the passage: 


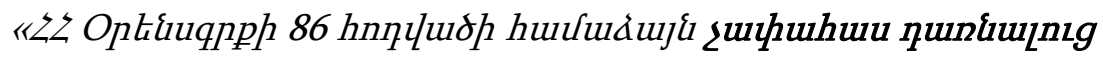

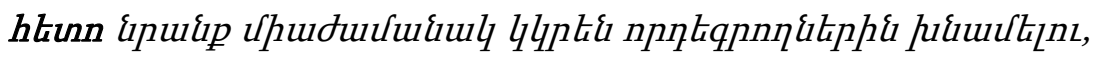

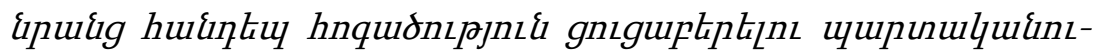
pjnukitip):

To solve this problem we can, for example, provide such a context which makes the meaning of the concept clear, using the method of descriptive translation or introducing a footnote (e.g., adult, i.e. more than 18 years old in Armenia and 20 years old in Canada).

\section{Conclusion}

Each country is characterized by a different linguistic, cultural and legal system. Therefore, legal translations entail a certain level of system specificity and are not challenging only from a linguistic point of view, but also from a legal one. The translator, as the recipient of the source text and the producer of the target text has to be fully aware of the referential processes and correlate the reference strategies of the two cultures in order to meet the requirements of a fully functional translation. Knowing the problem areas and pointing them out can help translators eliminate the risk of misunderstanding. Overall, a translator who specializes in legal translation, should have the ability to read with "legal expert eyes" and achieve conceptual adequacy adapting to the needs of specialized drafters and target readers, but also of non-specialized receivers, as appropriate, depending on the translation brief.

\section{References:}

1. Correia, R. (2003) "Translation of EU Legal Texts" in Crossing Barriers and Bridging Cultures: The Challenges of Multilingual Translation for the European Union. // Ed. by A. Tosi. Clevedon: Multilingual Matters Ltd.

2. Guranilk, D.B. (1979) Webster's New World Dictionary of the American Language. Cleveland, (Ohio): Collins.

3. Order N66 (07.12.2000) of RA Ministry of Social Security on Child's Adoption. Available at: <http://www.arlis.am/DocumentView.aspx?DocID= 
19374> [Accessed October 2017].

4. Pym, A. (2012) On Translator Ethics: Principles for Mediation Between Cultures. Amsterdam: Benjamins.

5. Šarčević, S. (2000) New Approach to Legal Translation. The Hague: Kluwer Law International.

6. Vermeer, H.J. (1996) A Skopos Theory of Translation: Some arguments for and against. Heidelberg.

7. Weston, M. (1991) An English Reader's Guide to the French Legal System. London: Berg.

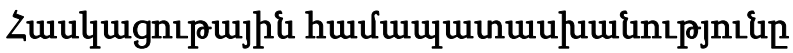

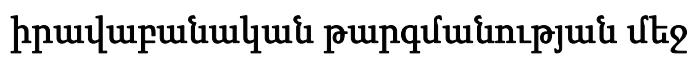

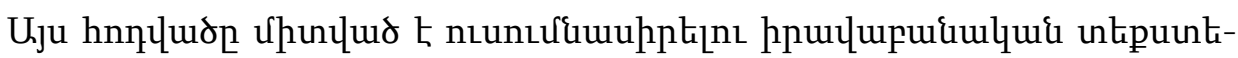

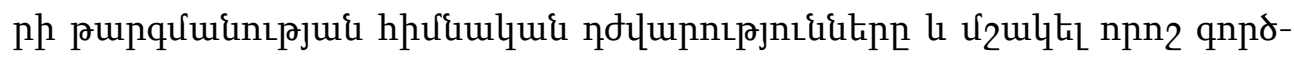

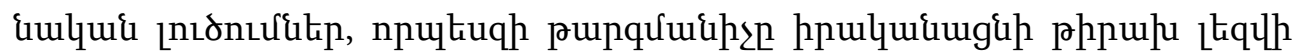

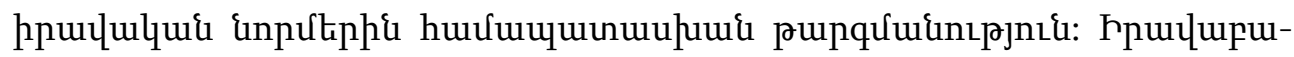

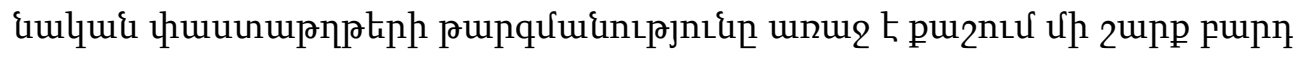

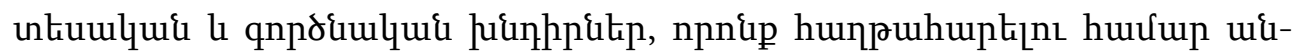

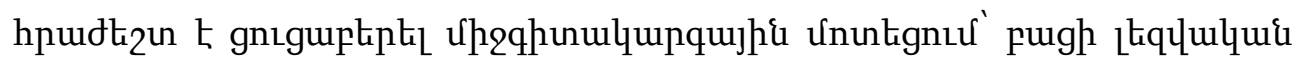

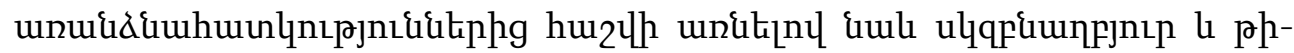

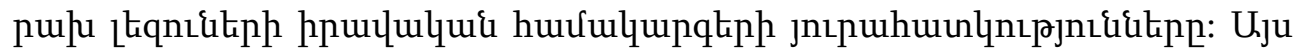

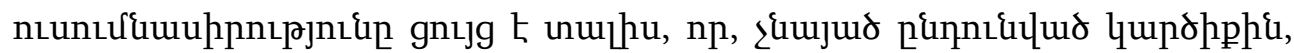

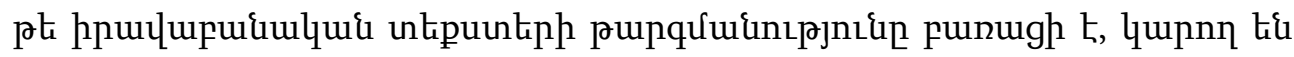

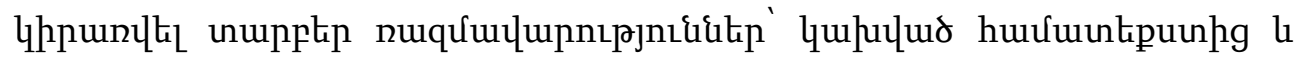

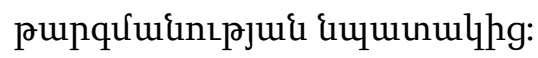

